\title{
JANIS: NEA JAva-based Nuclear Data Information System
}

\author{
Nicolas Soppera ${ }^{1, a}$, Manuel Bossant ${ }^{1}$, Oscar Cabellos ${ }^{1}$, Emmeric Dupont $^{2}$, and Carlos J. Díez ${ }^{1}$ \\ 1 OECD Nuclear Energy Agency, 92100 Boulogne-Billancourt, France \\ 2 CEA, Irfu, Universiteí Paris-Saclay, 91191 Gif-sur-Yvette, France
}

\begin{abstract}
JANIS (JAva-based Nuclear Data Information System) software is developed by the OECD Nuclear Energy Agency (NEA) Data Bank to facilitate the visualization and manipulation of nuclear data, giving access to evaluated nuclear data libraries, such as ENDF, JEFF, JENDL, TENDL etc., and also to experimental nuclear data (EXFOR) and bibliographical references (CINDA). It is available as a standalone Java program, downloadable and distributed on DVD and also a web application available on the NEA website. One of the main new features in JANIS is the scripting capability via command line, which notably automatizes plots generation and permits automatically extracting data from the JANIS database. Recent NEA software developments rely on these JANIS features to access nuclear data, for example the Nuclear Data Sensitivity Tool (NDaST) makes use of covariance data in BOXER and COVERX formats, which are retrieved from the JANIS database. New features added in this version of the JANIS software are described along this paper with some examples.
\end{abstract}

\section{Introduction}

JANIS [1] software is developed by the NEA Data Bank to facilitate the visualization and manipulation of nuclear data, giving access to evaluated data libraries, and to the EXFOR and CINDA databases [2]. It is available in several ways: as a standalone Java software, downloadable from the web and distributed on DVD and also a web application available on the NEA website. JANIS 4.0 was delivered in September 2013 [1] and new features described below will be available in JANIS 4.1.

A summary of JANIS databases and JANIS handbooks is presented in the paper. The procedure used to process evaluated data files into JANIS database is also described.

Recent NEA software developments rely on JANIS to access nuclear data such as covariances for the Nuclear Data Sensitivity Tool (NDaST) [3] and automated plots generation for the Nuclear Data Evaluation Cycle (NDEC) system [4]. The features recently added, notably automated plots generation through scripting via the command line and improvement of covariance data support are described, along with some example of the use of JANIS.

\section{JANIS databases}

Neutron evaluated libraries (e.g. TENDL-2015 [5]) are processed for being uploaded in JANIS Database (see Fig. 1) by merging ENDF-6 (original files) and PENDF (reconstructed-pointwise ENDF) files to produce HENDF (Hybrid ENDF) files, an acronym used at NEA, which includes reconstructed cross section from MF3, in consistency with MF33 covariance data when necessary, plus all the other MF data included in the original ENDF-6 format file.

Figure 2 presents the procedure to generate HENDF files. The processing code system used is essentially

a e-mail: Nicolas.SOPPERA@oecd.org
NJOY2012.50 [6] together with routines from the PREPRO and ENDF Checking \& Utility packages. The NJOY modules used in this processing are: MODER, RECONR, BROADR, HEATR, GASPR, ERRORR and COVR. For HENDF files, PENDF data are generated with general tolerance criteria for the reconstruction, thinning and broadening of $0.5 \%$, at $293.6 \mathrm{~K}$. The processed data contain kinematic kerma factors, total damage energy production and gas production cross section whenever such data are contained in the ENDF library. Processing covariance data into ERRORR and BOXER formats is carried out using SCALE-238 group structure.

Therefore, at the end of the processing three files are generated: one HENDF, one BOXER (if evaluated file provides covariance data) and one INTER (containing cross section integral data). These three files are then uploaded into JANIS database.

The content of all JANIS databases is summarized in Table 1.

NEA Data Bank is working on "Nuclear Data Evaluation Cycle" (NDEC) system [4], a systematized workflow for the verification, processing, differential validation and integral benchmarking of evaluated nuclear data. As one of its goal of taking advantage of synergies among NEA tools, NDEC has facilitated and automatized the processing of recent nuclear data libraries for JANIS database (e.g. TENDL-2015, FENDL-3.1b, BROND-3.1, IRDFF-1.05).

\section{JANIS Web}

The online nuclear data services are now provided through direct access to the JANIS databases taking advantage of the new Web interface of JANIS, which allows online browsing, searching and displaying nuclear data without requiring a Java Virtual Machine. The online services also include JANIS Books. 


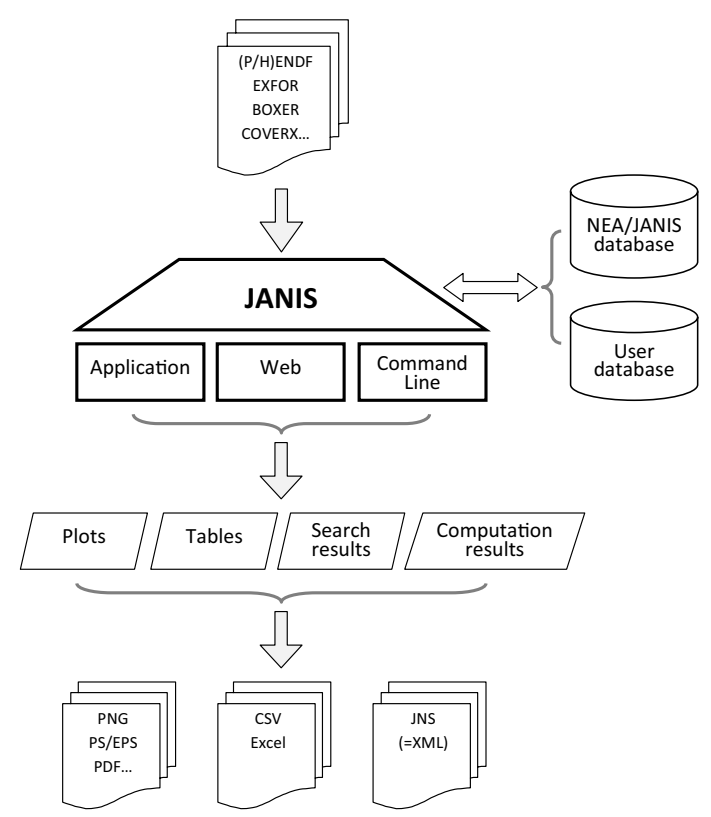

Figure 1. Database, capabilities and input/output information flow in JANIS.

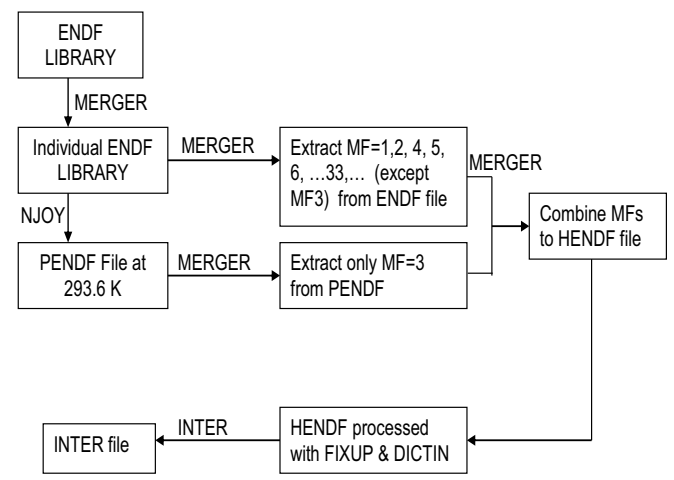

Figure 2. Flowchart of processing JANIS database from ENDF tapes.

The JANIS Books [1] are compilations of crosssection curves of evaluated and experimental data from a number of libraries, nuclear reactions and associated reaction products. JANIS Books are available for nuclear reactions induced by neutrons, photons and lightcharged particles. Online JANIS Books (JANIS web) are interactive versions that allow the users to zoom, access complementary information and plot additional data, as shown with the screenshot in Fig. 3 (e.g. ${ }^{238} \mathrm{U}$ total cross section). JANIS Books are also available for download in PDF format, showing in each page a cross section, with two plots plus integral values obtained from INTER.

JANIS can visualize different formats of uncertainties, both ENDF6/MF33 format and processed format, such as BOXER, ERRORR and COVERX. Recent new tools developed at NEA rely on JANIS features to access nuclear data, for example the Nuclear Data Sensitivity Tool (NDaST) which makes use of the BOXER and COVERX formats support and the JANIS database to retrieve covariance data.

\section{JANIS' new feature: The command line}

The JANIS command line was initially developed to satisfy specific users' requests. The basic syntax of the command
Table 1. Nuclear data libraries available in the NEA/JANIS database.

\begin{tabular}{|c|c|}
\hline nuclear properties & NUBASE-1997/2003/2012 \\
\hline radioactive data & $\begin{array}{c}\text { ENDF/B-VI.8/VII.0/VII.1, } \\
\text { GEFY-3.2/3.3/4.2/5.2, } \\
\text { JEF-2.2, JEFF-3.1/3.1.1, } \\
\text { JENDL-4.0, JENDL/FPD-2000, } \\
\text { JENDL/FPD-2011, JENDL/FPY-2011, } \\
\text { TENDL-2010 }\end{array}$ \\
\hline neutron data & $\begin{array}{c}\text { EXFOR, } \\
\text { BROND-2.2/3.1, } \\
\text { CENDL-2.1/3.1, } \\
\text { EAF-2007/2010, } \\
\text { ENDF/B-VI.8/VI.8-HE/VII.0/VII.1, } \\
\text { FENDL-2.1/2.1MG/3.1b, } \\
\text { GEFY-3.2/3.3/4.2/5.2/5.3, } \\
\text { IRDF-2002/2002MG, } \\
\text { IRDFF-1.0/1.0-640g/1.05, } \\
\text { JEF-2.2, } \\
\text { JEFF-3.0/3.0A/3.1/3.1.1/3.1.2/3.2, } \\
\text { JENDL-3.3/4.0, } \\
\text { JENDL/AC-2008, JENDL/FPY-2011, } \\
\text { JENDL/HE-2007, } \\
\text { RUSFOND-2010, } \\
\text { TENDL-2009 to TENDL-2015, } \\
\text { TSL ENDF/B-VI.8/VII.0, } \\
\text { TSL-JEFF3.0/3.1 }\end{array}$ \\
\hline gamma data & $\begin{array}{c}\text { EXFOR, } \\
\text { ENDF/B-VII.0/VII.1, } \\
\text { JENDL/PD-2004, } \\
\text { TENDL-2009 to TENDL-2015 }\end{array}$ \\
\hline proton data & $\begin{array}{c}\text { EXFOR, } \\
\text { ENDF/B-VI.8/VI.8-HE/VII.0/VII.1, } \\
\text { JEFF-3.1, } \\
\text { JENDL/HE-2007, JENDL-4.0, } \\
\text { PADF-2007, } \\
\text { TENDL-2009 to TENDL-2015 }\end{array}$ \\
\hline $\begin{array}{l}\text { deuteron and } \\
\text { triton data }\end{array}$ & $\begin{array}{c}\text { EXFOR, } \\
\text { ENDF/B-VI.8/ VII.0/VII.1, } \\
\text { TENDL-2009 to TENDL-2015 }\end{array}$ \\
\hline${ }^{3} \mathrm{He}$ data & $\begin{array}{c}\text { EXFOR, } \\
\text { ENDF/B-VII.0/VII.1, } \\
\text { TENDL-2009 to TENDL-2015 }\end{array}$ \\
\hline alpha data & $\begin{array}{c}\text { EXFOR, } \\
\text { JENDL/AN-2005, } \\
\text { TENDL-2009 to TENDL-2015 }\end{array}$ \\
\hline $\begin{array}{l}\text { heavy particles, } \\
\text { electron, } \\
\text { antiprotons, } \\
\text { kaons and pions }\end{array}$ & EXFOR \\
\hline
\end{tabular}

line is: "java -jar Janis.jar <options>". Most commands use JANIS identifiers to specify the data of interest (e.g. NEA N ENDF/B-VII.1 SIG U235 MT2 XS). The "-list" command gives the identifiers that can be used but these identifiers can also be inferred from either JANIS Web tree URLs, by replacing '/' with blanks and removing eventual single quotes, or from JNS XML files, by replacing ' ' with blanks. An example of command line to retrieve cross-section data in a table format is:

"java -jar Janis.jar -table NEA N JEFF-3.1.1 SIG U235 MT2")

The "-render" option was recently implemented to handle the conversion of JNS file into PNG graphics. 


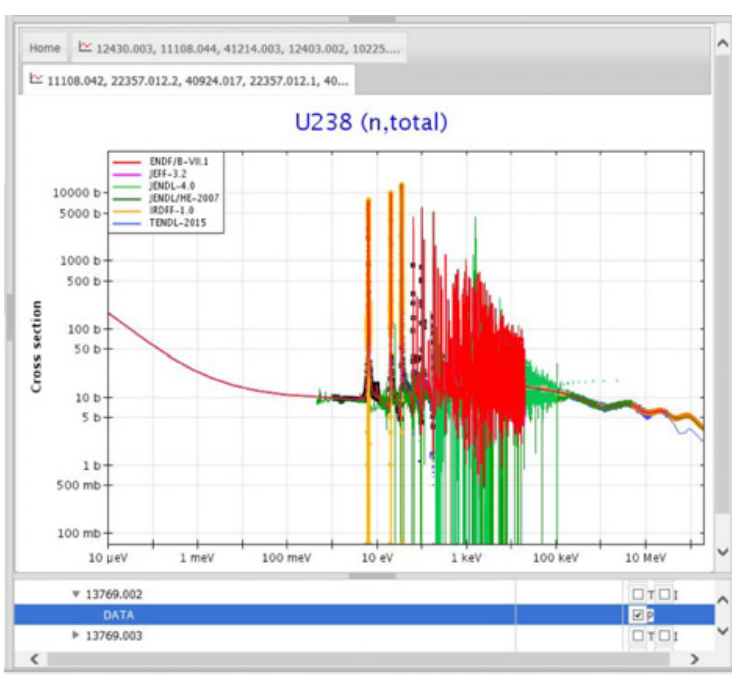

Figure 3. JANIS Books based on online JANIS version, no Java required (see http://www. oecd-nea.org/janisweb/).

JANIS-4.0 permits to save and restore the content of a renderer in an XML file (with extension "JNS", by default). It is available through the menu "File > Save..." and "File > Open...". The XML file includes all the information needed to re-open the renderer in the state it was saved. So, all data selected, including calculation results, and all customisation done on plots will be completely restored.

The new JANIS version allows automated plots generation (Fig. 4) through scripting via the command line execution: "java -jar Janis.jar -o FILE.png -render FILE.jns png 1024 768".

The command line option is also implemented in JANIS TRANS Checker tool which is used by EXFOR compilers to check the format of preliminary EXFOR files. This tool has been integrated in "EXFOR editor" software developed by Center of Nuclear Physics Data (CNPD), Russia, and also in the "EXFOR Compilation Tool" developed by Hokkaido University Nuclear Reaction Data Centre (JCPRG), Japan. See http://www.oecdnea.org/janis/trans-checker.html for more information.

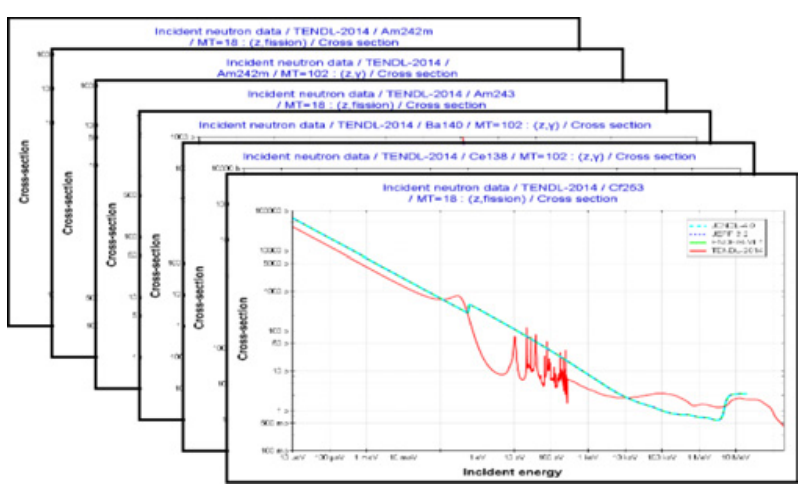

Figure 4. JANIS automated plots generation through scripting via command line.

\section{Conclusions and outlook}

JANIS has been developed to provide a user-friendly access to evaluated, experimental and bibliographic data available in ENDF and EXFOR/CINDA libraries. The NEA maintains the JANIS software and its database upto-date, while developing new features and capabilities after collecting information and feedback from a broad community of users: nuclear data experimentalists, EXFOR compilers, evaluators of nuclear data and users of nuclear data in many applications such as fission reactor neutronics, criticality, safety, waste management, fusion applications, material radiation damage...

An upcoming challenge will be to adapt to the successor of ENDF format which is currently being defined by the nuclear data community.

\section{References}

[1] N. Soppera, M. Bossant, E. Dupont, Nucl. Data Sheets 120, 294-296 (2014)

[2] N. Otuka et al. Nucl. Data Sheets 120, 272 (2014)

[3] J. Dyrda, this conference

[4] C.J. Díez, this conference

[5] A.J. Koning and D. Rochman, Nucl. Data Sheets 113, 2841 (2012)

[6] R.E. MacFarlane, D.W. Muir, R.M. Boicourt and A.C. Kahler, LA-UR-12-27079 Rev, LANL, 2015 